\title{
Driving Ability in Patients with Severe Chronic Low Back or Osteoarthritis Knee Pain on Stable Treatment with Tapentadol Prolonged Release: A Multicenter, Open-label, Phase 3b Trial
}

\author{
Rainer Sabatowski • Rüdiger Scharnagel • Anne Gyllensvärd • Ilona Steigerwald
}

To view enhanced content go to www.paintherapy-open.com Received: February 20, 2014 / Published online: April 4, 2014

(C) The Author(s) 2014. This article is published with open access at Springerlink.com

\section{ABSTRACT}

Introduction: Strong centrally acting analgesics, including tapentadol prolonged release (PR), have demonstrated efficacy for the management of non-malignant, chronic pain. Maintaining patient independence, including the ability to drive safely, is a key goal of long-term analgesic therapy. This multicenter, open-label, phase $3 \mathrm{~b}$ trial evaluated the effects of tapentadol PR on driving ability.

Methods: This study included patients who had completed previous tapentadol PR trials for

Trial registration: EudraCT 2009-015397-35.

Electronic supplementary material The online version of this article (doi:10.1007/s40122-014-0025-3) contains supplementary material, which is available to authorized users.

R. Sabatowski · R. Scharnagel

Comprehensive Pain Center, University Hospital

Carl Gustav Carus, Dresden, Germany

A. Gyllensvärd

Global Drug Safety, Grünenthal GmbH,

Aachen, Germany

I. Steigerwald $(\bowtie)$

Medical Affairs Europe and Australia,

Grünenthal GmbH, Aachen, Germany

e-mail: ilona.steigerwald@grunenthal.com severe low back or osteoarthritis pain. After at least 6 weeks of dose stability, patients continued taking tapentadol PR (50-250 mg twice daily) and could take supplemental immediate-release tapentadol $50 \mathrm{mg}$, except on the day before or day of the driving test (before the test). Pain intensity was assessed using an 11-point numerical rating scale. The Vienna Test System-Traffic Plus was used to assess cognitive and psychomotor function. The key surrogate parameter for driving ability was a global judgment based on 6 battery tests.

Results: Thirty-eight patients enrolled and completed the trial, and 35 patients completed all 6 tests. Pain scores remained unchanged from enrollment to final visit [mean (standard deviation) change, -0.2 (1.0)]. Approximately two-thirds $[65.7 \%(23 / 35)]$ of patients were classified as fit to drive based on the global judgment of driving-specific ability [34.3\% (12/ 35 ) not fit to drive]. Total daily tapentadol PR dose (>200 vs. $\leq 200 \mathrm{mg} /$ day) did not affect global judgment of driving ability $(P=0.4885)$. Two adverse events (considered unrelated to study drug) were reported.

Conclusion: Results suggest that most patients receiving a stable dose of tapentadol PR for 
severe, chronic pain would be able to drive, consistent with earlier studies evaluating stable treatment with strong opioids. Study design limitations and needs for individual patient assessment must be considered in clinical practice.

Keywords: Chronic pain; Driving ability; Opioids; Pain; Tapentadol prolonged release

\section{INTRODUCTION}

Strong centrally acting analgesics (e.g., opioids) are gaining acceptance for use in the management of non-malignant, chronic pain [1-3]. In addition to relieving pain, goals of long-term analgesic therapy are to allow patients to maintain their independence and stay active [4]. The ability to drive safely is a key component of daily living [4]. Studies have shown that patients on stable doses of opioid analgesics may be able to drive safely based on individual evaluations [5-10]. However, medications with $\mu$ opioid receptor agonist activity may adversely affect patients' cognitive and psychomotor performance, particularly during titration when dose changes occur [11-15].

Tapentadol is a centrally acting analgesic with two mechanisms of action, $\mu$-opioid receptor agonism and noradrenaline reuptake inhibition [16, 17]. Tapentadol prolonged release (PR) is approved in Europe for the management of severe chronic pain in adults, which can be adequately managed only with opioid analgesics [18], and in the United States (tapentadol extended release) for the management of moderate to severe, chronic pain, and neuropathic pain associated with diabetic peripheral neuropathy in adults when a continuous, around-the-clock opioid analgesic is needed for an extended period of time [19]. In a randomized, controlled phase 3 study [20], tapentadol PR (100-250 mg twice daily [bid]) was associated with a lower incidence of dizziness and fewer discontinuations due to nervous system side effects than oxycodone controlled release (20-50 mg bid). The lower incidence of nervous system side effects with tapentadol compared with oxycodone may be related to the contribution of noradrenaline reuptake inhibition to its analgesic activity $[17,20]$.

This multicenter, open-label, phase $3 \mathrm{~b}$ trial (EudraCT: 2009-015397-35) evaluated cognitive and psychomotor performance as measured by a validated methodology [21] (Vienna Test System-Traffic Plus; Schuhfried GmbH, Mödling, Austria) based on a global judgment as the key outcome surrogate parameter for driving ability in patients with severe, chronic low back pain or osteoarthritis knee pain on stable treatment with tapentadol PR. Based on prior results showing that patients on stable doses of opioid analgesics may be able to drive safely (depending on individual results) [5-10], we hypothesize that many patients receiving stable doses of tapentadol PR for severe, chronic pain will be considered fit to drive.

\section{METHODS}

This study was conducted between February 26, 2010 and September 3, 2010. Seven sites in Germany contributed patients for participation. Each site received approval of the study protocol from an Independent Ethics Committee. The test site (where the Vienna Test System-Traffic Plus was conducted) was the Comprehensive Pain Center at Carl Gustav Carus University Hospital of Dresden. All patients provided written informed consent before the start of any trial activity. 


\section{PATIENT POPULATION}

This study included men and women 18-70 years of age who had completed a previous clinical trial of tapentadol PR for the management of chronic low back or osteoarthritis pain [22, 23]. Patients were required to be fluent in German (based on the investigator's assessment) and taking an individually titrated (balancing efficacy and tolerability) dose of tapentadol PR (50-250 mg bid) that had been stable for $\geq 2$ weeks.

Patients with physical, neurological, or psychological disabilities, or symptoms that would interfere with the study assessment were excluded from the study. Women were excluded if they were pregnant or breastfeeding. Patients were also excluded if they had a history of allergy, hypersensitivity, or contraindications to tapentadol or its excipients, including acute or severe bronchial asthma or hypercapnia, or known or suspected paralytic ileus. Additional exclusion criteria included a known or suspected history of alcohol or drug abuse; a positive urine drug screen (except for opioids); severe renal impairment; moderately or severely impaired hepatic function; a history of seizure disorder or epilepsy; mild/moderate traumatic brain injury, stroke, transient ischemic attack, or brain neoplasm within the past year; or severe traumatic brain injury within the last 15 years.

The use of monoamine oxidase inhibitors was prohibited during the study; the use of alcohol on the day before the test visit and prior to completion of the Vienna Test System-Traffic Plus and the use of antihistamines on a regular basis were also prohibited. The following medications were prohibited for 14 days prior to enrollment and during the study if they had been verified to cause central nervous system side effects in an individual patient (based on information from the originating trial): anticholinergics, anticonvulsants, atypical antidepressants, selective serotonin reuptake inhibitors, tetracyclic and tricyclic antidepressants, antiemetics, antitussives, systemic bronchodilators, dopaminergic drugs, and neuroleptics; if the medication had been taken on a regular basis at a stable dose for at least 14 days prior to enrollment and had not caused central nervous system side effects, the patient could continue taking it.

\section{STUDY DESIGN}

This multicenter, open-label, phase $3 \mathrm{~b}$ trial included 3 periods: an enrollment period, a preparation and testing period, and a finalization period. The enrollment period (Day 1) coincided with the final visit of the previous trial $[22,23]$ or occurred within $12 \mathrm{~h}$ of the last intake of tapentadol PR in that trial. Upon enrollment, patients received a 30-day supply of tapentadol PR at their current dose, which was sufficient to cover treatment for the maximum period between enrollment and the final visit. Patients also received a 30-day supply of tapentadol immediate release (IR) $50 \mathrm{mg}$, which could be taken on demand ( $\leq 2$ doses/ day, $\geq 4 \mathrm{~h}$ apart; total tapentadol dose $\leq 500 \mathrm{mg}$ / day). No additional analgesic medication was allowed during the study.

During the preparation and testing period (between Day 2 and 21), patients continued to take tapentadol PR (50-250 mg bid). On the day of the test, a physical examination was performed, vital signs were measured, and a urine screening and an alcohol breath test were performed. Patients were then trained on the driving test system, and the test was administered. No tapentadol IR or alcohol consumption was allowed on the day before or the day of the driving test until after the test was completed. 
The finalization period (between Day 3 and 30) lasted from the day after the test until the final study visit. The final study visit could occur at any time after the test visit (e.g., if the test visit occurred on Day 2, the final visit could occur on Day 3). During the finalization period, patients continued to take tapentadol PR (50-250 $\mathrm{mg}$ bid) to ensure a stable dose regimen. All unused study drug was collected and all drug was accounted for at the final visit.

\section{STUDY EVALUATIONS}

Cognitive and psychomotor function was assessed using the Vienna Test System-Traffic Plus as a surrogate for evaluating driving ability under stable treatment conditions; the overall test system consists of ten single tests. The Vienna Test System-Traffic Plus is a validated electronic test battery [21], consisting of individual tests produced in up to 27 languages [24]. The test system includes a color monitor (and/or projector), speakers, a special keyboard with joysticks, steering wheels, two pedals, two peripheral displays with a light diode matrix, and the software. The test system provides an empirically validated model (called a "neuronal network"), which uses test scores to predict results of a standardized driving test. The model enables subjects to be assigned to an overall classification of driving-specific ability (specified as the global judgment of driving ability) based on the following six battery tests: the adaptive matrices test, the cognitrone, the tachistoskopic traffic conception test, the reaction test, the determination test, and the peripheral perception test. The global judgment of driving ability was used to determine whether a subject's psychomotor performance and cognition fulfill the criteria of driving a car safely. A binary (yes/no classification) outcome was created for each of the individual performance tests, with a successful outcome (i.e., positive response) defined as a score that was $\geq 16$ th percentile on the respective test (based on a normal distribution in healthy subjects) [10]. For the global judgment of driving ability, subjects who were considered fit to drive fell into one of the following three subgroups: subjects with adequate drivingrelated ability (score $\geq 16$ th percentile on all 6 battery tests), subjects with adequate driving ability and performance deficits that could be compensated (score $<16$ th percentile on $\geq 1$ of the battery tests, but performance in other areas is sufficient to compensate for performance deficits), or subjects with performance deficits that could be compensated to a limited extent (score $<16$ th percentile on $\geq 1$ of the battery tests, and test results do not conclusively demonstrate that subject's performance in other areas is sufficient to compensate for performance deficits). Subjects with performance deficits that could be compensated to a limited extent were only considered fit to drive if the scores obtained for the cognitrone, determination test, and tachistoskopic traffic conception tests were $\geq 16$ th percentile. Subjects who were considered not fit to drive fell into 1 of the following subgroups: subjects with performance deficits that could be compensated to a limited extent with a score $<16$ th percentile on $\geq 1$ of the cognitrone, determination test, or tachistoskopic traffic conception tests; noncompensable performance deficits (score $<16$ th percentile on $\geq 1$ of the battery tests, and adequate performance in other areas was not sufficient to compensate for performance deficits); or inadequate driving-related ability (score $<16$ th percentile on the majority of battery tests). 
The adaptive matrices test is a non-verbal test that requires subjects to identify the figurative pattern of a matrix and select the item from eight possible answers that completes the pattern; an estimate of general intelligence is produced based on the Rasch model according to the maximum likelihood method. The cognitrone requires the subject to compare an abstract figure with a model figure and determine the correspondence between the figures; the mean time to correct rejections is used as a measure of selective attention. For the tachistoskopic traffic perception test, subjects are shown pictures of traffic scenes ( $1 \mathrm{~s}$ each) and then asked to select items they recalled being in each picture from a list of 5 items; the number of lists answered correctly is used as a measure of perceptual speed. The reaction test, which measures decision speed and physical motor speed, involves presenting subjects with visual stimuli (colored circles) and auditory stimuli; the subject signals when a yellow circle and specific auditory signal appear in combination. The subject is asked to ignore all other auditory and visual signals; the mean reaction time serves as a measure of decision speed. In the determination test, subjects are required to identify various stimuli and react to those stimuli by pressing a corresponding response button on a panel (or pedal); the stimuli are presented slightly faster than would be optimal for the respondents' individual reaction speed, resulting in a condition of sensory stress. For this test, the number of correct reactions is used to assess the resilience of attention and reaction speed under sensory stress. The peripheral perception test is used to assess field of view and divided attention. In this test, subjects are asked to press a foot pedal when moving light stimuli (presented in the periphery of the subject's visual field) appear; at the same time, the subject is required to track a moving object and must therefore distribute their attention between the two tasks. In addition to these six battery tests, other components of the Vienna Test System-Traffic Plus that were evaluated included the Vienna Risk Taking Test-Traffic (assesses performance in a potentially dangerous traffic situation), the two-hand coordination test (measures sensorimotor coordination), the vigilance test (assesses attention in the form of sustained vigilance), and the visual pursuit test (assesses visual orientation ability and skill in gaining an overview).

Pain intensity was assessed on an 11-point numerical rating scale-3 (NRS-3; recalled average pain intensity score during the last 3 days from $0=$ "no pain" to $10=$ "pain as bad as you can imagine") at the enrollment and final visits. At the time of the test, patients also reported their current pain intensity (11-point NRS).

Safety evaluations included treatmentemergent adverse event (TEAE) reporting, vital sign evaluations, physical examinations, and a driving history questionnaire. All adverse events that occurred between the enrollment and final visits were defined as TEAEs. Vital signs, including systolic and diastolic blood pressure, pulse rate, and respiratory rate, were measured at the enrollment, test, and final visits. For patients with a valid driving license, the driving history questionnaire assessed the year of passing the driving test, class of driving license, approximate distance driven in the last year, and whether the patient felt fit to drive.

\section{STATISTICAL ANALYSES}

Based on exploratory studies of the effects of opioids on driving ability [9, 10], it was estimated that 30 patients would be sufficient to descriptively explore the effects of multiple doses of tapentadol PR on cognitive and 
psychomotor performance as surrogate parameters for driving ability. Pharmacodynamic and efficacy-related parameters were analyzed for all patients who completed the six battery tests of the Vienna Test System-Traffic Plus (the per protocol population). Safety analyses were performed for all patients who took $\geq 1$ dose of tapentadol PR or tapentadol IR (the safety population). All statistical analyses were performed using SAS Software (SAS Institute Inc., Cary, NC, USA).

The global judgment (an outcome surrogate parameter for driving ability) and outcomes for the individual performance tests were summarized descriptively using patient counts and percentages. Pain intensity scores at the enrollment, test, and final visits were summarized using descriptive statistics. TEAEs were summarized using the number and percentage of patients with adverse events by system organ class and preferred term.

Unlike comparable trials of strong opioids [9, 21, 25], this trial did not compare patients receiving tapentadol with a group of historical controls consisting of untreated healthy subjects. The Vienna Test System-Traffic Plus can use a reference population for internal comparison; because the test system has been validated against a standard driving test [21], no comparative arm was required. Threshold values for each test were defined as the 16th percentile of normally distributed test data from a representative age-independent sample that was transformed into a standard normal distribution.

Exploratory subgroup analyses were conducted to determine the impact of intelligence quotient (IQ; obtained from the adaptive matrices test results), age, educational level (captured as part of the Vienna Test System-Traffic Plus), daily dose of tapentadol
Table 1 Demographic characteristics (safety population)

\begin{tabular}{ll}
\hline Characteristic & All patients $(\boldsymbol{N}=\mathbf{3 8})$ \\
\hline Age, years & \\
Mean (SD) & $58.0(6.95)$ \\
Range & $44-69$ \\
Gender, $n$ (\%) & \\
Male & $14(36.8)$ \\
Female & $24(63.2)$ \\
Race, $n(\%)$ & $38(100)$ \\
White & \\
Body mass index, $\mathrm{kg} / \mathrm{m}^{2}$ & $32.0(6.71)$ \\
Mean (SD) &
\end{tabular}

$S D$ standard deviation

$\mathrm{PR}$, current pain intensity, and distance driven in the previous year on global judgment.

\section{RESULTS}

\section{Patients}

The safety population included 38 patients who were enrolled and completed the trial. Demographic data for those patients are summarized in Table 1. The per protocol population (patients who completed all 6 battery tests of the Vienna Test System-Traffic Plus) included 35 patients. Three patients in the safety population were excluded from the per protocol population because of major protocol violations. One of those patients had a history of alcohol abuse and impaired hepatic function due to formal alcohol abuse, both of which violated the study exclusion criteria. One patient took a prohibited medication (benzodiazepine), and one patient took tapentadol IR on the day of the test visit.

In the safety population, $86.8 \%(33 / 38)$ of patients held a valid driving license, and $78.9 \%$ 
(30/38) considered themselves fit to drive. The mean [standard deviation (SD)] estimated distance that patients with a valid driving license had driven in the previous year was $12,084.8(12,203.8) \mathrm{km}$ per patient.

\section{Treatment Exposure}

In the safety population, the mean (SD) duration of tapentadol PR exposure in the current study was 23.9 (7.92) days (range 8.0-43.0 days). The mean (SD) final stable tapentadol PR dose was 271.1 (113.68) $\mathrm{mg} /$ day (range 100.0-500.0 mg/day).

\section{Global Judgment of Driving Ability}

Approximately two-thirds [65.7\% (23/35)] of patients receiving tapentadol PR treatment were classified as fit to drive based on the global judgment. Of the 23 patients who were classified as fit to drive, 7 had adequate driving ability, 11 had performance deficits that could be compensated, and 5 had performance deficits that could be compensated to a limited extent. The five patients with performance deficits that could be compensated to a limited extent were classified as fit to drive because their relevant scores as defined in a previous study [10] for each single test (cognitrone, tachistoskopic traffic conception, and determination) were $\geq 16$ th percentile.

Twelve (34.3\%) patients were classified as not fit to drive. Of those patients, three had inadequate driving ability, seven had noncompensable performance deficits, and two had performance deficits that could be compensated to a limited extent. The two patients with performance deficits that could be compensated to a limited extent were classified as not fit to drive because their scores for $\geq 1$ single test (cognitrone,
Table 2 Vienna Test System-Traffic Plus individual test results (per protocol population; $N=35$ )

\begin{tabular}{|c|c|}
\hline Test & $\begin{array}{l}\text { Patients with } \\
\text { a positive } \\
\text { response, }{ }^{\mathrm{a}} n(\%)\end{array}$ \\
\hline Cognitrone & $35(100)$ \\
\hline Vienna Risk-Taking Test-Traffic & $35(100)$ \\
\hline Tachistoskopic traffic conception test & $33(94.3)$ \\
\hline $\begin{array}{l}\text { Vigilance-mean value reaction time } \\
\text { correct hits }\end{array}$ & $32(91.4)$ \\
\hline Vigilance-number of correct hits & $31(88.6)$ \\
\hline Vigilance-number of incorrect hits ${ }^{b}$ & $30(85.7)$ \\
\hline Determination test & $30(85.7)$ \\
\hline Visual pursuit test & $29(82.9)$ \\
\hline $\begin{array}{l}\text { Peripheral perception test-field of } \\
\text { vision }\end{array}$ & $28(80.0)$ \\
\hline $\begin{array}{l}\text { Peripheral perception test-tracking } \\
\text { deviation }\end{array}$ & $27(77.1)$ \\
\hline Two-hand coordination test & $25(71.4)$ \\
\hline Reaction test (mean reaction time) & $23(65.7)$ \\
\hline Reaction test (mean motor time) & $20(57.1)$ \\
\hline Adaptive matrices test & $11(31.4)$ \\
\hline
\end{tabular}

a A positive response was defined as a test result $\geq 16$ th percentile of the normally distributed test data from a representative age-independent sample

b Fewer incorrect hits is associated with a better score

tachistoskopic traffic conception, and/or determination) were $<16$ th percentile [10].

\section{Overview of Individual Test Results}

Individual test results from the Vienna Test System-Traffic Plus are summarized in Table 2. More than $70 \%$ of patients were classified as having a successful outcome for the cognitrone, tachistoskopic traffic conception, determination, peripheral perception (field of vision and tracking deviation), Vienna Risk Taking Test-Traffic, two-hand coordination, 
Table 3 Global judgment ratings by IQ, distance driven in the previous year, age, education level, TDD of tapentadol $\mathrm{PR}$, and current pain intensity

\begin{tabular}{lcc}
\hline Variable & Fit to drive, $\boldsymbol{n}$ & $\begin{array}{l}\text { Not fit to } \\
\text { drive, } \boldsymbol{n}\end{array}$ \\
\hline IQ & & \\
$<85$ & 14 & 10 \\
$\geq 85$ & 9 & 2
\end{tabular}

Distance driven in the previous year $(\mathrm{km})$

$\begin{array}{lrr}\leq 9,000 & 8 & 8 \\ >9,000 & 12 & 2 \\ \text { Age (years) } & & \\ \leq 58 & 17 & 3 \\ >58 & 6^{\mathrm{a}} & 9\end{array}$

Education level $^{\mathrm{b}}$

$\begin{array}{rrr}\leq 3 & 18 & 1 \\ >3 & 5 & \end{array}$

TDD of tapentadol PR

$\begin{array}{rrr}\leq 200 \mathrm{mg} & 12 & 8 \\ >200 \mathrm{mg} & 11 & 4 \\ \text { Current pain intensity } & \end{array}$

\begin{tabular}{rrr}
$\leq 3$ & 15 & 10 \\
$>3$ & 8 & 2 \\
\hline
\end{tabular}

$I Q$ intelligence quotient, $P R$ prolonged release, $T D D$ total daily dose

a $P=0.0107$ vs. patients $\leq 58$ years of age (in favor of patients $\leq 58$ years of age)

${ }^{\mathrm{b}}$ For educational level, a level of $\leq 3$ indicates no school degree or secondary school or vocational training completed, and a level of $>3$ indicates completion of higher school or a university degree

c Current pain intensity was rated at the test visit on an 11-point numerical rating scale $(0=$ "no pain" to $10=$ "pain as bad as you can imagine")

vigilance, and visual pursuit tests. Between 50 and $70 \%$ of patients had successful reaction tests (mean reaction time and mean motor time). A total of $31.4 \%(11 / 35)$ of patients had a successful adaptive matrices test.

\section{Exploratory Analyses of Variables Affecting Global Judgment}

Exploratory subgroup analyses were conducted to determine the impact of IQ, educational level, age, daily dose of tapentadol PR, current pain intensity, and distance driven in the previous year on global judgment (Table 3). Patients with an IQ $\geq 85$ generally performed better in the test system and were more fit to drive than patients with an $\mathrm{IQ}<85$, but the differences were not statistically significant ( $P=0.2590$, Fisher's exact test). Patients who had driven $>9,000 \mathrm{~km}$ in the previous year tended to have better global judgment than those who had driven $\leq 9,000 \mathrm{~km}(P=0.0577$, Fisher's exact test). Patients $>58$ years of age performed significantly worse and were less fit to drive than those $\leq 58$ years of age $(P=0.0107, \quad$ Fisher's exact test). The following variables had no effect on global judgment assessments of driving ability: educational level $[\leq 3$ (no degree, secondary school, or vocational training) vs. $>3$ (higher school or university degree); $P=0.6399$; Fisher's exact test], total daily dose of tapentadol PR $\quad(>200$ vs. $\leq 200 \mathrm{mg} /$ day; $P=0.4885$; Fisher's exact test), and current pain intensity $[<3$ vs. $\geq 3$ (NRS-3); $P=0.4340$, Fisher's exact test].

\section{Efficacy-Related Parameter}

Pain scores on the 11-point NRS remained relatively unchanged from the start to the end of the trial, indicating that the pain relief achieved during the previous phase $3 \mathrm{~b}$ studies $[22,23]$ was maintained with continued tapentadol PR treatment during this trial. Pain intensity scores are summarized in Table 4. 
Table 4 Pain Intensity Scores (Per Protocol Population) ${ }^{\mathrm{a}}$

\begin{tabular}{lllll}
\hline & $\begin{array}{l}\text { Enrollment visit } \\
(\boldsymbol{n}=\mathbf{3 5})\end{array}$ & $\begin{array}{l}\text { Test visit } \\
(\boldsymbol{n}=\mathbf{3 5})\end{array}$ & $\begin{array}{l}\text { Final visit } \\
(\boldsymbol{n}=\mathbf{3 3})\end{array}$ & $\begin{array}{l}\text { Change from enrollment } \\
\text { to final visit }(\boldsymbol{n}=\mathbf{3 3})\end{array}$ \\
\hline Mean (SD) & $2.7(1.27)$ & $2.6(1.63)$ & $2.5(1.62)$ & $-0.2(1.0)$ \\
Median & 3.0 & 3.0 & 2.0 & 0.0 \\
Range & 0.0 to 6.0 & 0.0 to 6.0 & 0.0 to 6.0 & -2.0 to 2.0 \\
\hline
\end{tabular}

NRS numerical rating scale, $S D$ standard deviation

${ }^{a}$ Pain intensity scores at the enrollment and final visits were the average pain intensity scores during the last 3 days (11-point NRS-3); the score at the test visit was current pain intensity (11-point NRS)

\section{Safety}

Non-serious TEAEs were reported by $5.3 \%(2 / 38)$ of patients. One patient reported nasopharyngitis and one patient had a contusion of the rib cage; both of these TEAEs were considered by the investigator to be unrelated to study drug. No serious TEAEs, deaths, or discontinuations due to adverse events were reported. There were no notable changes observed in vital signs, except for increases in blood pressure and pulse rate during the test visit. During the test visit, mean (SD) systolic blood pressure was 10.3 (15.50) $\mathrm{mmHg}$ higher, diastolic blood pressure was $11.2(11.14) \mathrm{mmHg}$ higher, and pulse rate was 9.4 (18.56) beats per minute higher compared with the enrollment visit. These changes were not considered to be medically relevant.

\section{DISCUSSION}

Based on the global judgment, results of this trial show that patients under treatment with a stable dose of tapentadol PR (50-250 mg bid) for the management of non-malignant, chronic low back pain or osteoarthritis knee pain do not have clinically significant impairment of psychomotor or cognitive function that would prevent them from performing complex daily activities, such as driving a car. All individual tests of the Vienna Test System-Traffic Plus supported the results of the global judgment, except for the adaptive matrices test.

The driving ability of patients under stable tapentadol PR treatment in this trial is comparable to that observed in previous studies in patients under stable treatment with transdermal fentanyl [10] or transdermal buprenorphine [9]. Results of those studies [9, 10] showed that the driving ability of patients under stable transdermal treatment with fentanyl or buprenorphine was non-inferior to that of healthy, untreated subjects, indicating that patients on stable treatment with strong opioids might be able to drive safely based on individual evaluations. The percentage of patients $(34.3 \%)$ in the current trial with an individual result $\geq 16$ th percentile for 5 key tests (cognitrone, tachistoskopic traffic conception, determination, vigilance, and two-hand coordination tests) was comparable to or higher than the percentage of patients with an individual result $\geq 16$ th percentile in those previous studies with transdermal fentanyl [10] or transdermal buprenorphine [9].

Exploratory subgroup analyses showed that educational level, the total daily dose of tapentadol PR, and current pain intensity at 
the test visit had no effect on driving ability assessments. Patients in this study population were on a stable dose of tapentadol PR and their pain was well controlled at enrollment, as indicated by the low mean pain intensity score (2.7). At these stable doses of tapentadol PR, pain control was maintained throughout the study, with mean pain intensity scores of 2.6 at the test visit and 2.5 at final evaluation. Patients with a higher IQ $(\geq 85)$ and those who had driven greater distances in the previous year $(>9,000 \mathrm{~km})$ tended to perform better in the test system and be fit to drive, although these differences did not reach statistical significance. A significant difference was observed in driving ability between older ( $>58$ years of age) and younger patients. These results are not unexpected, given the previously demonstrated effect of age on driving ability in a general population of individuals holding driving licenses [26]. Previous results have shown poorer performance for older drivers (60-82 years of age) compared with middleaged drivers (40-50 years) [26] in measures of driving ability, including performance on a traffic-related tachistoskopic perception test and the amount of time needed in tracking and reaction tests.

There were potential limitations to this study that should be considered. The global judgment results should be interpreted with caution due to the exploratory nature of the analyses. The use of a historical comparison, rather than healthy, untreated controls, should also be considered. Nevertheless, the Vienna Test System-Traffic Plus has been validated against a standard driving test and can use a reference population for internal comparison [21]. In addition, no pre-study measurements of driving ability were performed, so a causal treatment effect on patients with a negative global judgment (i.e., those that were considered not fit to drive) could not be determined; future studies should assess driving ability before and after tapentadol treatment initiation. This study, like the majority of studies evaluating effects of opioids on cognitive function (including driving ability), is also limited by the duration of the study and does not report on long-term treatment effects [27]. However, unlike other studies, in the current study patients were on drug for 12 weeks and on stable treatment for at least 6 weeks prior to entering the trial as they had previously participated in another clinical, well-documented good clinical practice trial (ClinicalTrials.gov Identifiers: NCT00982280, NCT00986258, NCT00983073, or NCT00983385 [22, 23] ).

Analgesic therapy for the management of non-malignant, chronic pain may not only provide adequate pain relief, but may also allow patients to preserve their independence and stay active, including maintaining the ability to drive safely [4]. Pain of high intensity can reduce cognitive capacity and impair driving performance [28, 29]; when pain intensity is reduced, patients may be better able to focus on driving. Consistent with results of the current study, numerous studies have shown that patients on stable doses of opioid analgesics may be able to drive safely based on individual evaluations [5-10]. Epidemiologic evidence also indicates that patients on stable doses of opioid analgesics are not at an increased risk for being in fatal or non-fatal car accidents [6].

\section{CONCLUSION}

Results of this trial based on a global judgment parameter suggest that most patients under treatment with a stable dose of tapentadol PR 
(50-250 mg bid) for the management of severe chronic pain would be able to drive. Outcomes of the current study are overall consistent with earlier studies $[9,10]$, supporting driving ability for patients under stable treatment with strong opioids. Methodological limitations (e.g., lack of pre-study measurement of driving ability) need to be taken into account when interpreting these results. Individual responses to treatment with tapentadol PR may vary, and individual driving assessments for patients who are prescribed tapentadol should be considered in cases of uncertainty for an individual patient (e.g., patients with additional risk factors, such as impaired cognitive or psychomotor performance). In general, the safety profile of tapentadol PR observed in this study was consistent with that observed in previous multiple-dose studies of tapentadol PR in patients with non-malignant, chronic pain $[20,22,23,30-32]$.

\section{ACKNOWLEDGMENTS}

This study and article processing charges were sponsored by Grünenthal $\mathrm{GmbH}$, Aachen, Germany. Editorial support for the writing of this manuscript was provided by Cherie Koch, PhD, of MedErgy, and was funded by Medical Affairs, Grünenthal GmbH, Aachen, Germany. The authors retained full editorial control over the content of the manuscript. All named authors meet the ICMJE criteria for authorship for this manuscript, had full access to all of the data in this study, take responsibility for the integrity of the work as a whole, and have given final approval for the version to be published. Results of this study were presented in part at the International Association for the Study of Pain (IASP) 14th World Congress on Pain, August 27-31, 2012, Milan, Italy.
Conflict of interest. Rainer Sabatowski has received funds as a principal investigator from Grünenthal $\mathrm{GmbH}$, Allergan, and Astella and has received speaker's honoraria from MSD and Janssen-Cilag GmbH. Anne Gyllensvärd is an employee of Grünenthal GmbH. Ilona Steigerwald is an employee of Grünenthal GmbH. Rüdiger Scharnagel declares no conflict of interest.

Compliance with ethics guidelines. All procedures followed were in accordance with the ethical standards of the responsible committee on human experimentation (institutional and national) and with the Helsinki Declaration of 1975, as revised in 2000 and 2008. Informed consent was obtained from all patients for being included in the study.

Open Access. This article is distributed under the terms of the Creative Commons Attribution Noncommercial License which permits any noncommercial use, distribution, and reproduction in any medium, provided the original author(s) and the source are credited.

\section{REFERENCES}

1. Kalso E, Allan L, Dellemijn PL, et al. Recommendations for using opioids in chronic non-cancer pain. Eur J Pain. 2003;7:381-6.

2. Sullivan MD, Edlund MJ, Fan MY, Devries A, Brennan BJ, Martin BC. Trends in use of opioids for non-cancer pain conditions 2000-2005 in commercial and Medicaid insurance plans: the TROUP study. Pain. 2008;138:440-9.

3. Zhang W, Moskowitz RW, Nuki G, et al. OARSI recommendations for the management of hip and knee osteoarthritis, Part II: OARSI evidence-based, expert consensus guidelines. Osteoarthr Cartil. 2008;16:137-62.

4. Kress HG, Kraft B. Opioid medication and driving ability. Eur J Pain. 2005;9:141-4. 
5. Byas-Smith MG, Chapman SL, Reed B, Cotsonis G. The effect of opioids on driving and psychomotor performance in patients with chronic pain. Clin J Pain. 2005;21:345-52.

6. Fishbain DA, Cutler RB, Rosomoff HL, Rosomoff RS. Can patients taking opioids drive safely? A structured evidence-based review. J Pain Palliat Care Pharmacother. 2002;16:9-28.

7. Gaertner J, Radbruch L, Giesecke T, et al. Assessing cognition and psychomotor function under longterm treatment with controlled release oxycodone in non-cancer pain patients. Acta Anaesthesiol Scand. 2006;50:664-72.

8. Mailis-Gagnon A, Lakha SF, Furlan A, Nicholson K, Yegneswaran B, Sabatowski R. Systematic review of the quality and generalizability of studies on the effects of opioids on driving and cognitive/ psychomotor performance. Clin J Pain. 2012;28:542-55.

9. Dagtekin O, Gerbershagen HJ, Wagner W, Petzke F, Radbruch L, Sabatowski R. Assessing cognitive and psychomotor performance under long-term treatment with transdermal buprenorphine in chronic noncancer pain patients. Anesth Analg. 2007;105:1442-8.

10. Sabatowski R, Schwalen S, Rettig K, Herberg KW, Kasper SM, Radbruch L. Driving ability under longterm treatment with transdermal fentanyl. J Pain Symptom Manage. 2003;25:38-47.

11. Hill JL, Zacny JP. Comparing the subjective, psychomotor, and physiological effects of intravenous hydromorphone and morphine in healthy volunteers. Psychopharmacology. 2000;152:31-9.

12. Kerr B, Hill H, Coda B, et al. Concentration-related effects of morphine on cognition and motor control in human subjects. Neuropsychopharmacology. 1991;5:157-66.

13. Bruera E, Macmillan K, Hanson J, MacDonald RN. The cognitive effects of the administration of narcotic analgesics in patients with cancer pain. Pain. 1989;39:13-6.

14. Friswell J, Phillips C, Holding J, Morgan CJ, Brandner B, Curran HV. Acute effects of opioids on memory functions of healthy men and women. Psychopharmacology. 2008;198:243-50.

15. Kamboj SK, Tookman A, Jones L, Curran HV. The effects of immediate-release morphine on cognitive functioning in patients receiving chronic opioid therapy in palliative care. Pain. 2005;117:388-95.
16. Tzschentke TM, Christoph T, Kögel B, et al. (-)-(1R,2R)-3-(3-dimethylamino-1-ethyl-2-methylpropyl)-phenol hydrochloride (tapentadol $\mathrm{HCl}$ ): a novel m-opioid receptor agonist/norepinephrine reuptake inhibitor with broad-spectrum analgesic properties. J Pharmacol Exp Ther. 2007;323:265-76.

17. Tzschentke TM, De Vry J, Terlinden R, et al. Tapentadol hydrochloride. Analgesic, mu-opioid receptor agonist, noradrenaline reuptake inhibitor. Drugs Future. 2006;31:1053-61.

18. Palexia ${ }^{\circledR}$ SR (tapentadol) prolonged-release oral tablets [summary of product characteristics]. Buckinghamshire: Grunenthal Ltd.; 2011.

19. Nucynta ${ }^{\circledR}$ ER (tapentadol) extended-release oral tablets C-II [package insert]. Raritan: Janssen Pharmaceuticals, Inc.; 2011.

20. Buynak R, Shapiro DY, Okamoto A, et al. Efficacy and safety of tapentadol extended release for the management of chronic low back pain: results of a prospective, randomized, double-blind, placeboand active-controlled Phase III study. Expert Opin Pharmacother. 2010;11:1787-804.

21. Risser R, Chaloupka C, Grundler W, Sommer M, Hausler J, Kaufmann C. Using non-linear methods to investigate the criterion validity of trafficpsychological test batteries. Accid Anal Prev. 2008;40:149-57.

22. Steigerwald I, Muller M, Kujawa J, Balblanc J-C, Calvo-Alen J. Effectiveness and safety of tapentadol prolonged release with tapentadol immediate release on-demand for the management of severe, chronic osteoarthritis-related knee pain: results of an open-label, phase $3 \mathrm{~b}$ study. J Pain Res. 2012;5:121-38.

23. Steigerwald I, Muller M, Davies A, et al. Effectiveness and safety of tapentadol prolonged release for severe, chronic low back pain with or without a neuropathic pain component: results of an open-label, phase 3b study. Curr Med Res Opin. 2012;28:911-36.

24. Schuhfried. Internationalization. http://www. schuhfried.com/vienna-test-system-vts/internation alization/. Accessed 17 March 2014.

25. Shmygalev S, Damm M, Weckbecker K, Berghaus G, Petzke F, Sabatowski R. The impact of long-term maintenance treatment with buprenorphine on complex psychomotor and cognitive function. Drug Alcohol Depend. 2011;117:190-7.

26. Schlag B. Elderly drivers in Germany-fitness and driving behavior. Accid Anal Prev. 1993;25:47-55. 
27. Kendall SE, Sjogren P, Pimenta CA, Hojsted J, Kurita GP. The cognitive effects of opioids in chronic noncancer pain. Pain. 2010;150:225-30.

28. Kuhajda MC, Thorn BE, Klinger MR, Rubin NJ. The effect of headache pain on attention (encoding) and memory (recognition). Pain. 2002;97:213-21.

29. Veldhuijzen DS, van Wijck AJ, Wille F, et al. Effect of chronic nonmalignant pain on highway driving performance. Pain. 2006;122:28-35.

30. Afilalo M, Etropolski MS, Kuperwasser B, et al. Efficacy and safety of tapentadol extended release compared with oxycodone controlled release for the management of moderate to severe chronic pain related to osteoarthritis of the knee: a randomized, double-blind, placebo- and activecontrolled phase III study. Clin Drug Investig. 2010;30:489-505.

31. Schwartz S, Etropolski M, Shapiro DY, et al. Safety and efficacy of tapentadol ER in patients with painful diabetic peripheral neuropathy: results of a randomized-withdrawal, placebo-controlled trial. Curr Med Res Opin. 2011;27:151-62.

32. Wild JE, Grond S, Kuperwasser B, et al. Long-term safety and tolerability of tapentadol extended release for the management of chronic low back pain or osteoarthritis pain. Pain Pract. 2010;10:416-27. 